\title{
Fluorescence In Vivo Endomicroscopy Part 2: Applications of High-Resolution, 3-Dimensional Confocal Laser Endomicroscopy
}

\section{Mohammedayaz Rangrez, ${ }^{1}$ Lindsay Bussau, ${ }^{2 *}$ Kishwar Ifrit, ${ }^{2}$ Mark C. Preul, ${ }^{3}$ and Peter Delaney ${ }^{4}$}

${ }^{1}$ Optiscan, Toronto, Ontario, Canada

${ }^{2}$ Optiscan Pty Ltd., Mulgrave, Victoria, Australia

${ }^{3}$ Barrow Neurological Institute, Phoenix, Arizona

${ }^{4}$ Orb-Med Consulting, Melbourne, Victoria, Australia

*lbussau@optiscan.com

\begin{abstract}
Fluorescence in vivo endomicroscopy (FIVE) is a state-ofthe-art endoscopy technique used to image tissue interactions and molecular events in a cell. In Part 1 of this series, the history, types of confocal laser endomicroscopy (CLE), and limitations of the technology were discussed. In Part 2 of the series, we describe several applications of FIVE technology, including advances in cancer, gastrointestinal, liver, rectal mucosal barrier function, acute lung injury, and brain imaging. Future perspectives for the technology are also presented.
\end{abstract}

Keywords: in vivo endomicroscopy, fluorescence, cancer, acute lung injury, brain imaging

\section{Introduction}

In Part 1 of this series, we described fluorescence in vivo endomicroscopy (FIVE) technology and some of the advantages and limitations of the instruments [1]. In simple terms, it is a laser scanning confocal microscope where the imaging end is attached to an optical fiber allowing the operator to "peek" inside a living organism. Confocal laser endomicroscopy (CLE) has reversed the age-old approach where the biological tissue was resected, processed, stained, and imaged on a microscope stage. With FIVE, tissue architecture can be imaged instantaneously with the use of intravenous (i.v.) and topical fluorescent dyes, providing a virtual real-time biopsy. FIVE is a cutting-edge CLE technology with subcellular resolution and optical sectioning competency. Compared to other CLEs, FIVE visualizes three-dimensional (3-D) tissue architecture in vivo and provides information on tissue interactions with its environment and even molecular events in the cell (Figure 1). It has successfully achieved the vision of endoscopists to obtain histoarchitecture of a given tissue while performing endoscopy. Over the last 20 years, FIVE has been used in integrated confocal endomicroscopes for targeted gastrointestinal biopsies, in a neurosurgical device for surgical assistance in brain cancer surgery, and for veterinary and laboratory imaging purposes. Several applications and the future perspectives of FIVE are described below.

\section{Fluorescence In Vivo Endomicroscopy Applications}

Intraoperative imaging for surgical guidance in cancer. The goal of cancer surgery is complete extirpation of diseased tissue. The challenge in meeting this requirement is the accurate determination of the extent of tumor spread and infiltration of apparent margins. The current gold standard, histopathological examination of excised tissue, provides cellular detail but has several drawbacks. Firstly, quick histopathological confirmation (frozen sections) takes between 20 minutes and up to an hour while the surgeon waits for the pathologist's interpretation. Frozen section results are generally considered to be of lower accuracy when compared to more time-consuming methods but are nonetheless crucial for the rapidity with which cellular information can be gleaned. For fixed tissues, pathology may take up to a week for results. Secondly, the excised tissue shrinks after excision while the wound area expands. Thus, it is extremely difficult to pinpoint an area of the wound bed by looking at the excised tissue. The surgeon must often guess and rely on his/her experience, if the pathologist recommends cutting more from a specific area of the wound bed. Thirdly, this process does not provide the surgeon cellular-level detail while performing the surgery. Critical tumor resection surgeries can be lifethreatening and can leave the patient physically impaired if too much tissue is excised. FIVE provides a suitable alternative to histopathological examination during cancer surgery. It can capture images while the surgery is being performed, rapidly providing the surgeon with cellular details of the tissue to be excised. FIVE can be used with fluorophores showing tissue architecture, or with cancer specific fluorophores, and can provide the surgeon confirmation and confidence on the excision procedure.

FIVE has been used successfully for performing intraoperative virtual biopsies. Nguyen and his colleagues [2] performed FIVE imaging in patients who underwent surgery for intraabdominal malignancy (gastric adenocarcinoma, pancreatic ductal adenocarcinoma, and small bowel lymphoma) for realtime, intraoperative, in vivo histologic surface and subsurface imaging. The surgeons reported fluorescein contrast enabled visualization of cellular histoarchitecture and epidermal morphology of visceral organs, with magnification and resolution equivalent to light microscopy. This enabled distinction between normal and diseased tissue and influenced surgical decisions. Similarly, Preul and his team tested the usability of FIVE in 74 glioma and meningioma patients. First-generation FIVE devices were able to detect gliomas with $94 \%$ specificity and $91 \%$ sensitivity and meningiomas with $93 \%$ specificity and $97 \%$ sensitivity [3]. FIVE can also work as a molecular imaging tool to visualize epidermal growth factor receptors (EGFR) in cell culture, mice xenograft models, and human biopsied tissue 


\section{TESCAN Dynamic micro-CT}

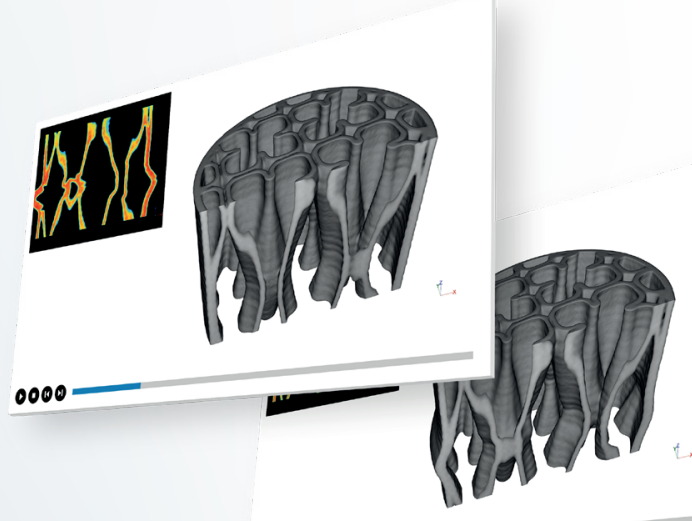

Leading the way in temporal resolution shifting your research from 3D to 4D

0000

Dynamic Imaging of Aluminum Foam Compression - 20 sec/scan, 190 total scans

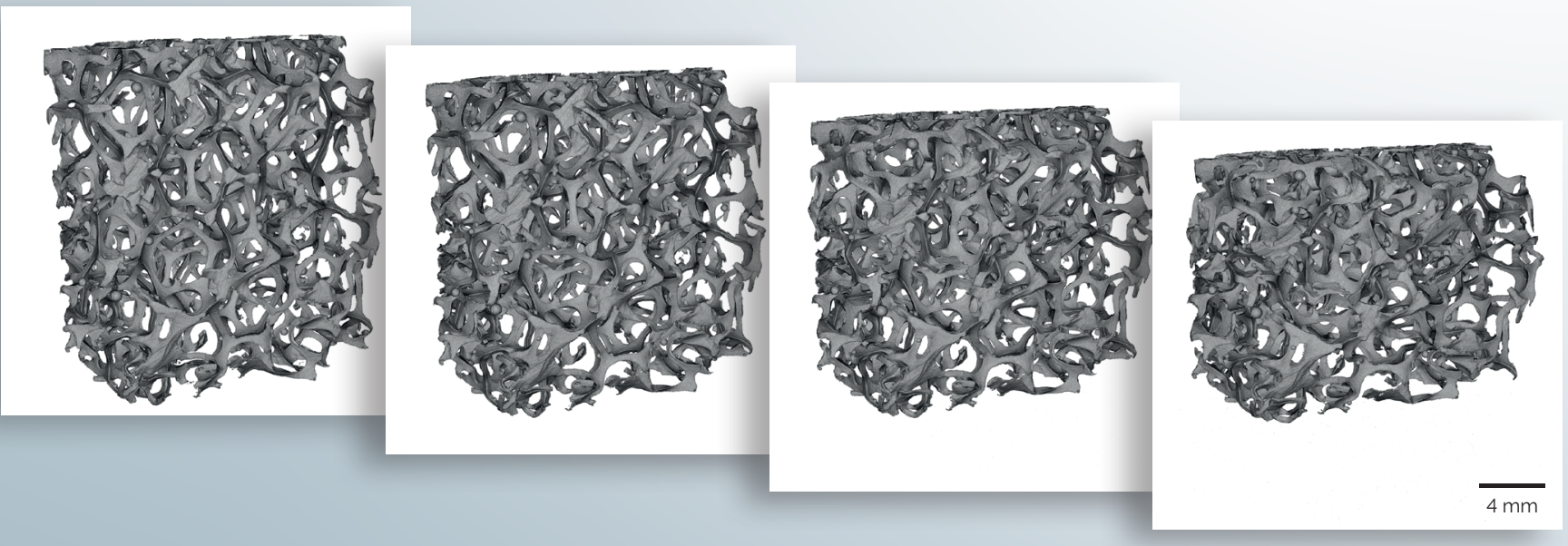

Dynamic Imaging of Dissolving Soap

Bubbles - $30 \mathrm{sec} / \mathrm{scan}, 150$ total scans

Analysis of Soap Bubble Coalescence
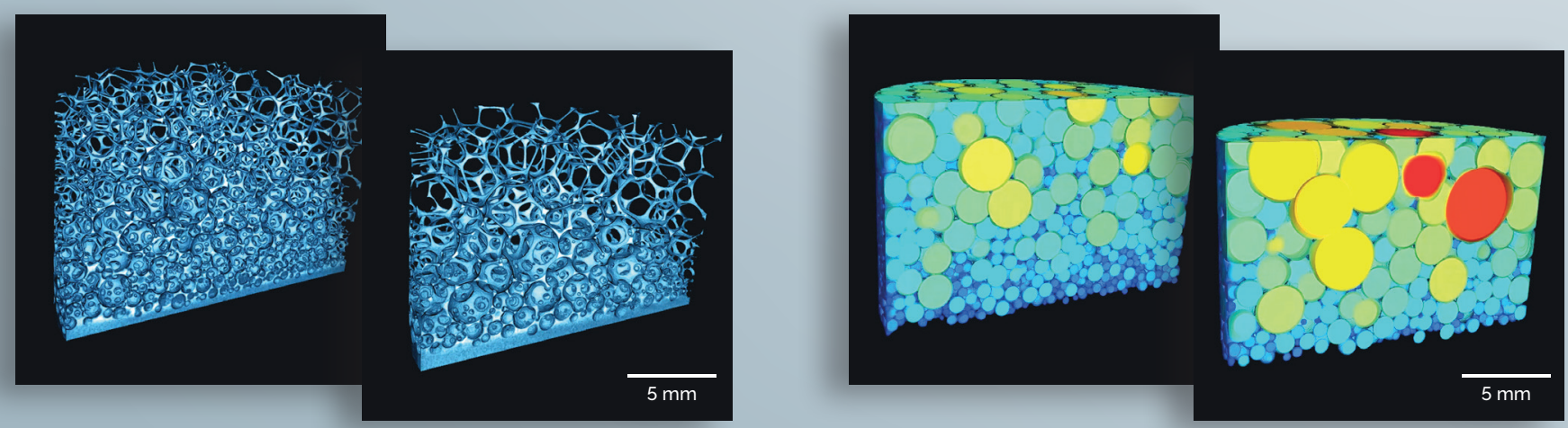

What can you do with Dynamic CT?

Z̈TESCAN

Contact us today to find out.

wwW.tescan.com

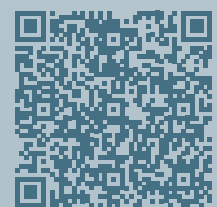




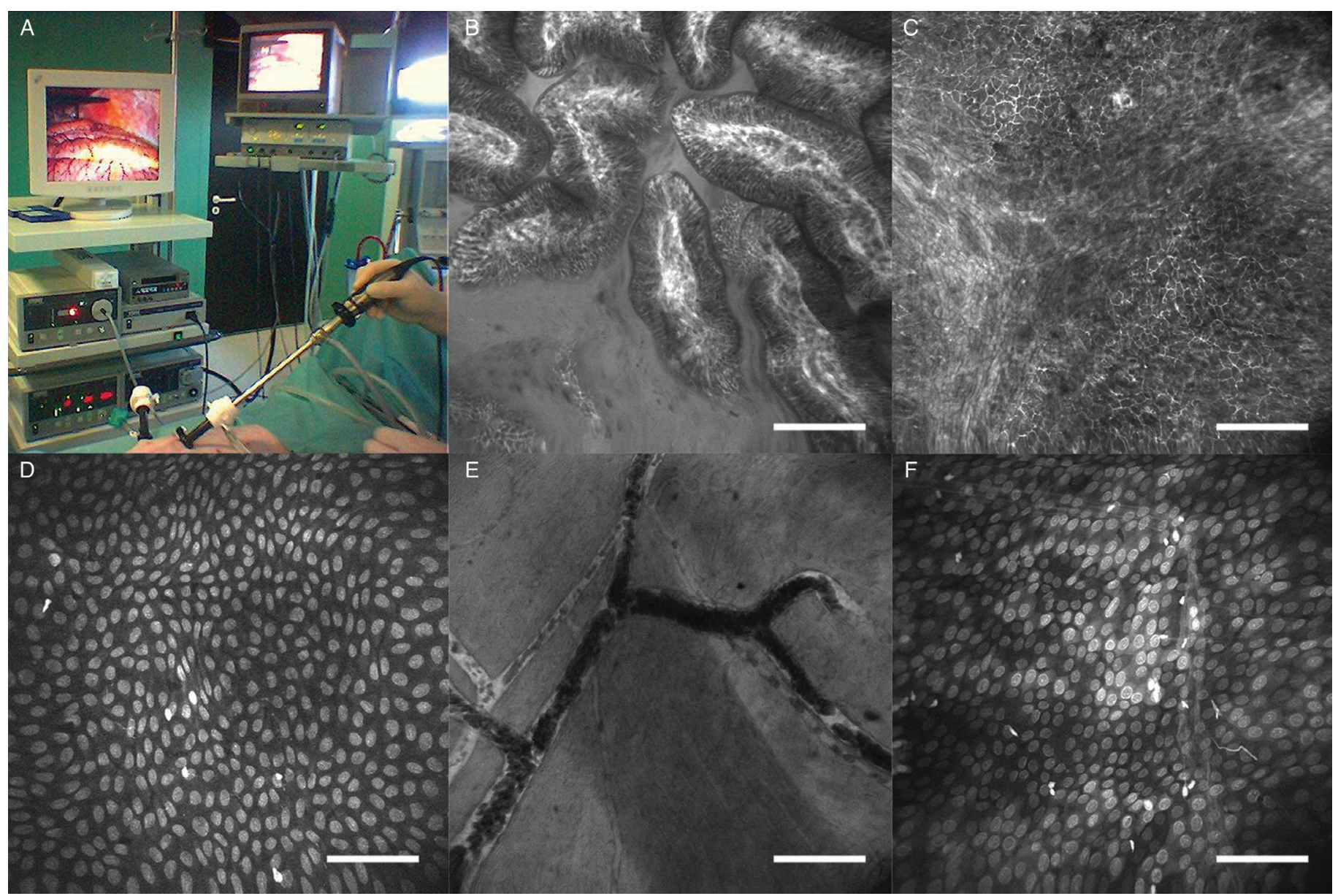

Figure 1: Laparoscopic imaging of a pig with FIVE. (A) Instrument setup. (B) Histoarchitecture of microvilli in the ileum. Contrast agent, i.v. fluorescein. (C) Microvasculature of the liver. Contrast agent, i.v. fluorescein. (D) Liver capsule. Contrast agent, topical $0.5 \%$ acriflavine. (E) Blood vessels imaged from the serosal surface of the bladder. Contrast agent, i.v. fluorescein. (F) Perineum. Contrast agent, topical $0.5 \%$ acriflavine. Scale bars $=100 \mu \mathrm{m}$.

with fluorophore tagged EGFR antibodies [4]. FIVE imaging confirmed various degrees of EGFR expression in different cell lines, as well as in living mice. Similar studies targeting vascular endothelial growth factor (VEGF) in colorectal cancer has also been reported, suggesting that FIVE can contribute to the identification of lesions and predict response to targeted treatment [5]. Moreover, these studies also demonstrated the use of FIVE as an in vivo immunohistochemical/molecular imaging apparatus in preclinical studies.

Gastrointestinal imaging. FIVE has been extensively used in imaging gastrointestinal tissue [6-8] and clinically proven to successfully characterize Barrett's esophagus [9], gastritis [10], Helicobacter pylori infection [11], intestinal metaplasia [12], gastric hyperplastic and adenomatous polyps [13], colon polyps, and rectal cancers [14-15]. Many clinical studies have reported FIVE-integrated endomicroscopes (also known as Pentax ISC-1000 and eCLE) as a highly accurate tool for detection of Barrett's esophagus [16] and neoplasia, greatly reducing the needed number of biopsies [17-19]. Though other CLEs can also detect Barrett's esophagus, FIVE has higher inter-rater agreement and accuracy [20]. Many other studies have also reported the efficiency of FIVE in detecting Barrett's esophagus and esophageal cancer as well as imaging its virtual histology [21-22]. Similarly, FIVE offers great potential in surveillance of patients having diffuse gastric mucosal alterations, with high prevalence of gastric cancer where random biopsies are suboptimal [23]. FIVE has been extensively used for detecting gastric cancer with $99 \%$ accuracy in diagnosis of high-grade neoplasia and superficial cancer [24]. This study also reported differentiated gastric cancerous mucosa with hypervascularity and various caliber microvessels with irregular shapes. Undifferentiated gastric cancers disclosed hypovascularity and irregular, short branch vessels. In addition to detecting gastric cancers, FIVE was also efficient in detecting residual cancer following endoscopic submucosal dissection [25]. Similar to clinical studies, FIVE has been employed for imaging gastric mucosa in research dogs [26], providing high-quality histology equivalent images of gastric mucosa, and allowing the assessment of cytoplasmic and microvascular features using i.v. fluorescein and topical application of acriflavine (Figure 2). These authors were also able to diagnose and monitor infection of canines with Helicobacter and monitored the effectiveness of treatment.

FIVE has also been used for imaging intestinal mucosa. In fact, serendipitous observations, made in patients with endomicroscopy, have answered key questions and spawned 


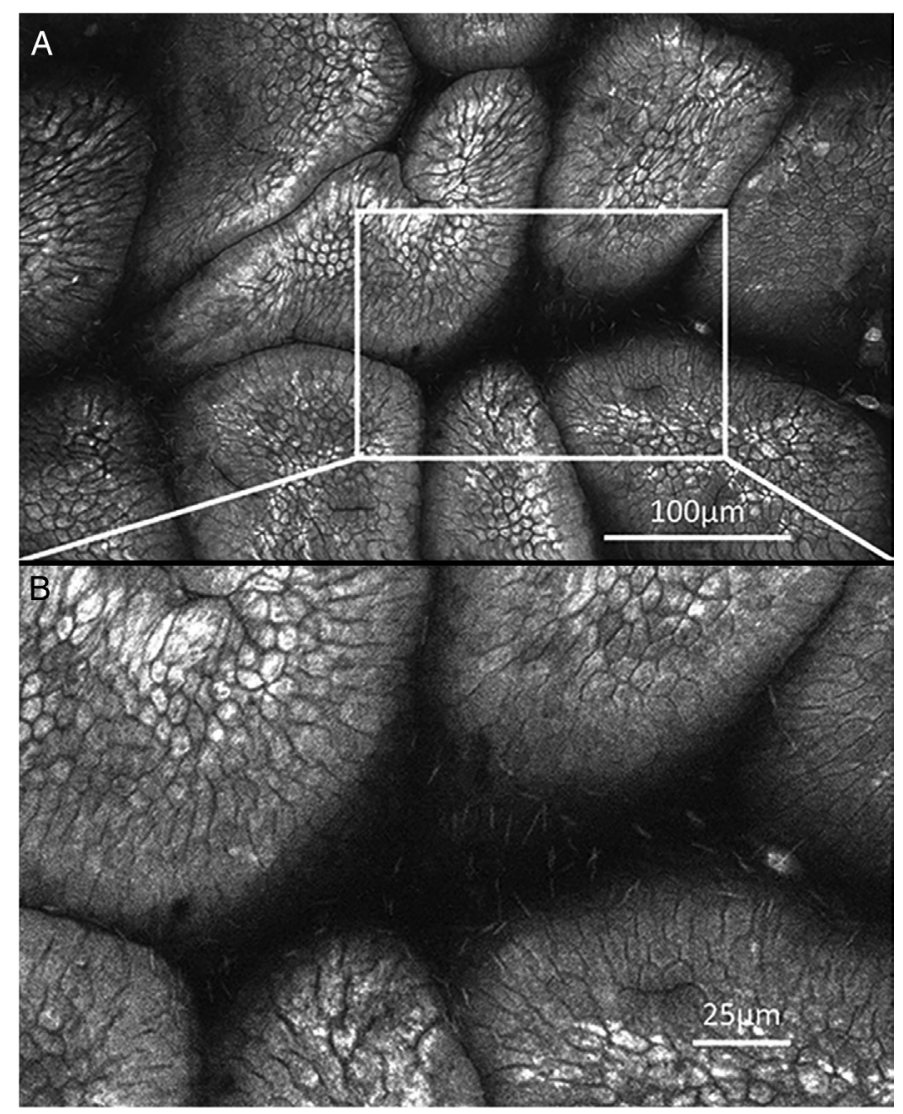

Figure 2: Cellular detail captured in the gastrointestinal tract with FIVE. (A) Dog gastric mucosa stained with $0.5 \%$ acriflavine. (B) Canine helicobacter in mucus surrounding gastric villus. Images published with permission from Dr. M. Sharman, Faculty of Veterinary and Agricultural Science, University of Melbourne.

significant lines of investigation relating to epithelial cell turnover and gut barrier function. During the establishment of gastrointestinal applications of FIVE, small bright structures were commonly observed in the single columnar epithelial cell layer. These were initially dismissed as artifacts, or "junk" tissue fragments absorbing fluorescein. However, investigators noted that single epithelial cells pool fluorescein, burst, and are ejected from the epithelium (Figure 3). Further, it was noted that the surrounding epithelial cells joined basally, thus maintaining barrier function. Over just a few minutes, this was observed to happen repeatedly in various parts of the epithelium - a constant process of stunningly rapid and frequent cell shedding. This led to multiple investigations of aberrations to this process in patients suffering inflammatory bowel disease (IBD) and molecular studies in animals which were then refined to human patients. It has since been quantified and found predictive of recurrent flares in patients with quiescent inflammatory conditions [27]. This reset of our understanding has clarified the dynamics of gut cell turnover and barrier function. The surprising impact of these observations highlight the importance of observing cellular events in vivo.

Kiesslich imaged intestinal mucosa after staining with acriflavine in 17 patients, obtaining 6,277 images from human terminal ileum and rectum [28]. Mucosal imaging with FIVE has also been demonstrated for diseases like gastrointestinal mucosa-associated lymphoid tissue (MALT) lymphoma, where results are in almost perfect agreement with histological findings [29]. Out of 26 cases reported with histology, only one case of deep tissue lymphoma was not diagnosed with FIVE, as a limitation of FIVE is that it cannot access structures deep in the tissue. FIVE has also been used for imaging graft versus host disease (GvHD) [30], celiac disease [27], restorative proctolectomy [31], inflammatory bowel disease [32], and ulcerative colitis [33]. In a study focused on epithelial cell permeability and mucosal healing, FIVE was used for imaging small intestinal permeability using fluorescein. High intestinal permeability, confirmed through FIVE imaging, is highly correlated with altered lipid metabolism, heightened innate immune response, and junctional protein signaling [34]. FIVE imaging has also been used for imaging colorectal cancer, as it can detect precursor colorectal polyps and predict intraepithelial neoplasia with $99.1 \%$ accuracy [35]. In a parallel study, Kiesslich evaluated intraepithelial neoplasia and colorectal cancer with acriflavine and fluorescein and found FIVE to be rapid, accurate, and highly predictive of cancer [36]. Many other studies have also reported sensitivity, specificity, and accuracy of FIVE in colorectal cancer detection $[14,15,37]$.

FIVE offers dynamic preclinical animal imaging, as one can use brighter fluorophores with higher specificity and lower toxicity. A mouse study comparing FIVE with two-photon microscopy (2P) showed that both achieved $176 \mu \mathrm{m}$ penetration depth and could identify goblet cells [38]. In a similar study, cresyl violet-based staining was used to stain the ileum and colon of BL-6 mice [39]. Similarly, intestinal imaging in experimental pericardial tamponade in anesthetized Vietnamese mini pigs demonstrated injury to intestinal mucosa [40]. FIVE has also been used to image temporal progression of 2,4,6-trinitrobenzenesulfonic acid-induced acute colitis and its treatment with oral phosphatidylcholine (PC) in rats. Damage included serosal microcirculation and structural injury, including disruption of the capillary network [41]. Varga found similar results, including changes in epithelial structure, microvasculature, and inflammation [42].

Barrier function studies. Vargas reported a lack of tools for evaluation of rectal mucosal barrier function and explored FIVE for assessing in vivo structural and functional barrier integrity [43]. Topical and i.v. fluorescent probes provided subcellular resolution of the mucosal surface and assessment of barrier function loss following topical application of a microbicidal agent. Injury due to the microbicides compromised mucosal barrier function of the rectum, increasing the risk for transmission of HIV and other pathogens. Though H\&E staining could not offer functional or temporal imaging, it did confirm the in vivo findings of barrier compromise. These reports suggest FIVE as an effective tool for detecting epithelial injury and barrier loss, offering real-time rectal mucosal evaluation.

Pharynx and pulmonary imaging. Haxel examined various regions of the anterior oropharynx in patients, collecting real-time in vivo and ex vivo cellular and subcellular imaging with topical acriflavine and i.v. fluorescein as contrast agents [44]. FIVE imaging provided details of the surface epithelium, 


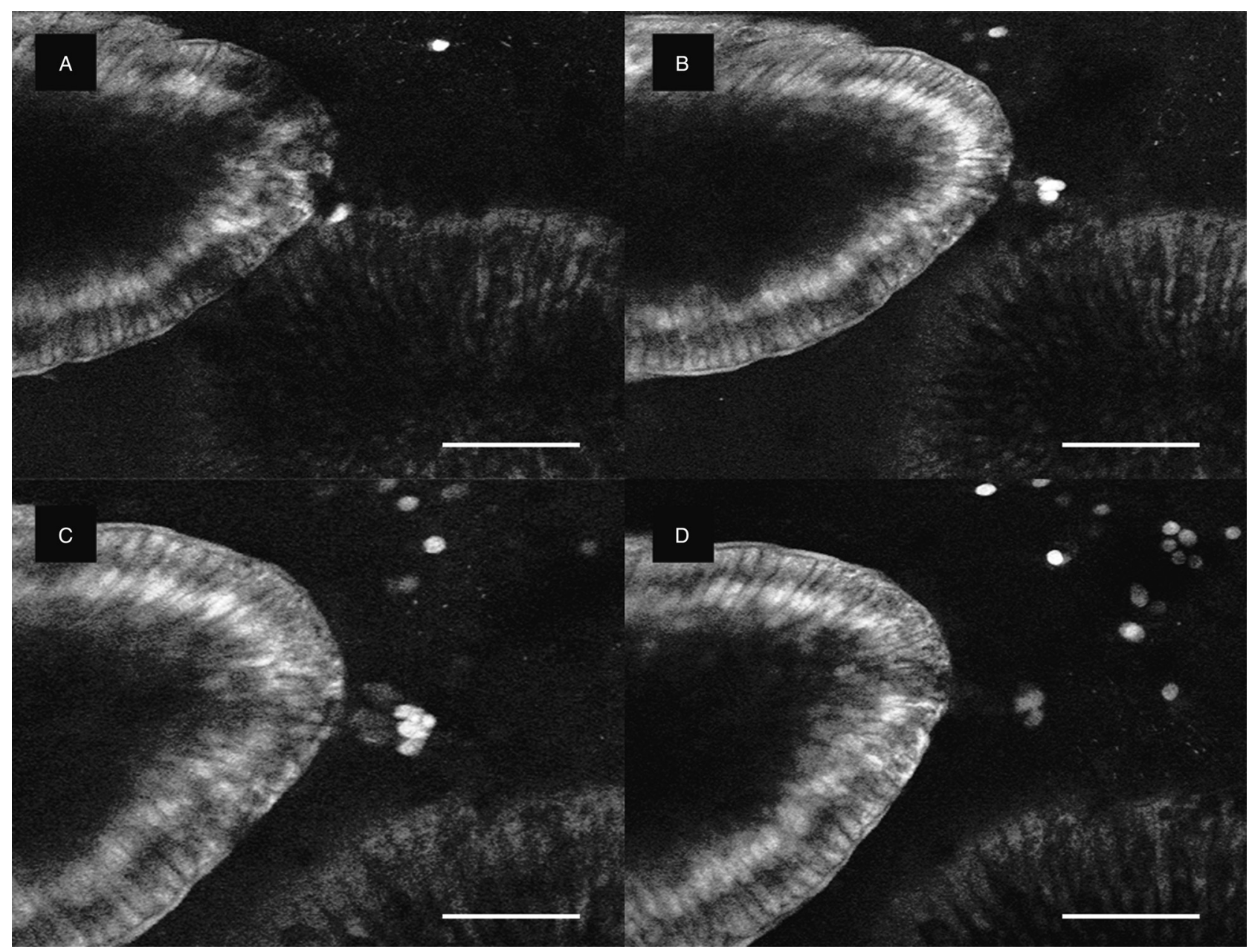

Figure 3: Sequential images captured in real time during ongoing endoscopy of a single villus in the patient's terminal ileum after i.v. administration of the fluorophore fluorescein sodium. The process of shedding a single epithelial cell is observed over a period of 1-2 min. (A) The epithelial cells of an intestinal villus begin form singular "gaps," and there is an accumulation of fluorescein around neighboring cells. (B) An epithelial cell is ejected leaving a small plume of fluorescein, and the adjacent cells are beginning "zip up" the gap, restoring barrier function first from the basement membrane toward the luminal side. (C) The adjacent cells have almost formed a uniform arrangement. Further closing the gap. (D) Barrier function appears to be restored as the necessary cell-to-cell junctions consolidate. Images courtesy of Prof. Dr. Ralf Kiesslich, medical director Helios Dr. Horst Schmidt Clinics, Wiesbaden; and Prof. Dr. med. Martin Götz, Chief Physician Gastroenterology / Oncology, Sindelfingen-Böblingen Clinic, Boeblingen. Scale bars $=100 \mu \mathrm{m}$.

subepithelial layers, and mucosal capillary network. Ex vivo staining with acriflavine-stained epithelial cell nuclei and the histoarchitecture correlated well with the histology of biopsy specimens. Musani studied normal human airway histology and endobronchial adenocarcinoma and compared FIVE with a traditional surgical histopathology approach. Images captured from the trachea, primary and secondary carinae, and endobronchial mass showed cellular and subcellular structures of the respiratory mucosa and submucosa, and allowed imaging of pseudostratified columnar epithelium, lamina propria, and microvasculature that distinguished normal airway epithelium and malignant tissue [45].

Acute lung injury (ALI) and acute respiratory distress syndrome (ARDS) are life-threatening conditions characterized by severe acute hypoxemic respiratory failure and bilateral lung infiltrates [46]. ALI/ARDS must be differentiated from other acute pulmonary disease entities, but there are no "easy-to-do" diagnostic tests. The gold standard is open lung biopsy, which has a relatively low benefit/harm ratio [47]. In theory, FIVE can provide cellular and structural assessment of living tissue using a small confocal probe in direct contact with the visceral pleura, and this theory was tested in a rat model of lung injury [48]. FIVE facilitated visualization of the microcapillary network around alveoli, epithelial cell membranes, lung cell apoptosis, neutrophil extravasation, and pulmonary edema. These results were confirmed with ex vivo fluorescent methods (Figure 4) and suggested FIVE as a promising tool for improving bedside diagnostic and decision-making therapeutic strategy in patients with ALI. Studies of CLE, including FIVE, for lung imaging was reviewed by Lesur [49].

Brain imaging. Fluorescence-based imaging techniques are emerging as valuable tools in neurosurgery to highlight vascular structures and tumors (Figure 5). In a study of patients with brain neoplasm, FIVE was used intraoperatively after injecting i.v. fluorescein. A total of 20,734 CLE images were correlated with 267 biopsy specimens, and FIVE specificity and sensitivity were $94 \%$ and $91 \%$ for gliomas and $93 \%$ and 97\% for meningiomas [3]. In a similar study, FIVE imaging in 


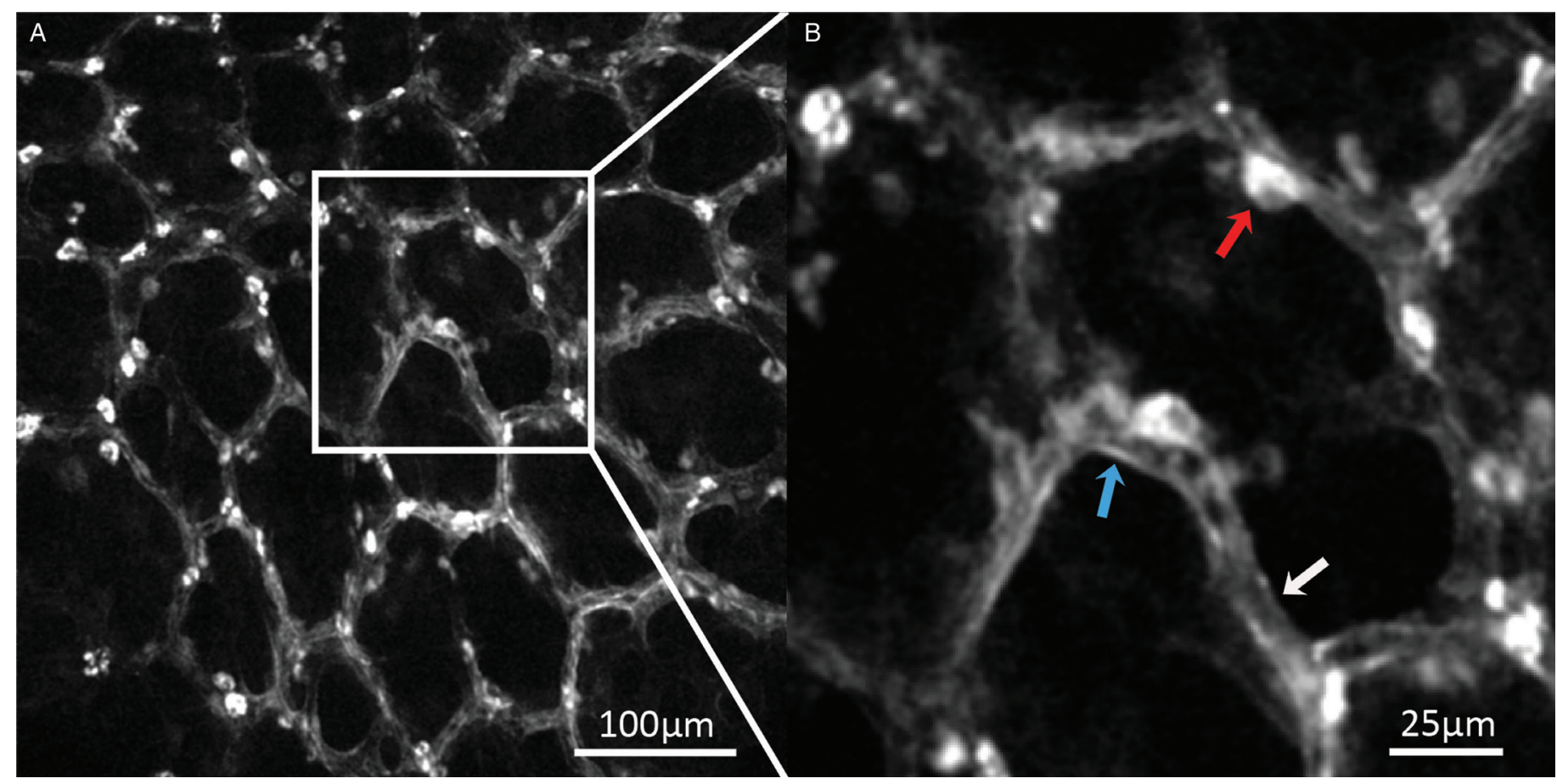

Figure 4: (A) Histoarchitecture of rat lung stained with acridine orange, $0.05 \%$ i.v. Ricinus communis lectin in bleomycin induced lung injury model. (B) Enhanced parenchymal thickness (blue and white arrows) together with macrophage and metaplastic epithelial cell "big cell" staining (red arrow). Image published with permission from Dr. Olivier Lesur, University of Sherbrooke, Canada.

patients undergoing brain tumor surgeries was used to evaluate volumetric images of cytoarchitecture and the course of abnormal vasculature in 31 human tumors, including meningiomas, gliomas, and pituitary adenomas [50]. These findings provided increased spatial understanding of tumor cellular architecture and related structures compared with two-dimensional (2-D) classical procedures. FIVE has an added advantage, as digital biopsy allows real-time transfer of images from the operating room to virtually anywhere in the world. An FDA-approved clinical FIVE neurosurgical system (CONVIVO) has been used intraoperatively to examine brain tumor biopsied $e x$ vivo (Figure 6). Trials currently have been extended in several clinical centers to assess the feasibility of the system for in vivo neurosurgical application, which are showing promising integration into the neurosurgical-pathology workflow.

Parallel with their clinical findings, Belykh et al. also described FIVE imaging in mouse brain and confirmed the histoarchitecture in 3-D volumetric examination [50]. Optical sectioning with FIVE allowed extensive visualization of tumor size, shape, location, and orientation. In a similar glioblastoma study of murine brain cell shape and tissue architecture, Sankar reported well-correlated FIVE and histology images [51]. Peyre also imaged mouse brain meningioma using FIVE, capturing accurate imaging of meningothelial and fibroblastic mouse meningiomas, as well as malignant meningiomas that corresponded with pathological findings [52]. This study also showed a sharp definition of the brain-tumor interface and enabled identification of embedded nerves, vessels, and an extension of tumor along Virchow-Robin spaces into adjacent tissue. A similar study by Fenton et al. reported Alexa Fluorlabeled, tyrosine-related protein-2 antibodies for imaging mouse glioma cells [53]. Martirosyan et al. extended their acridine orange histoarchitecture glioma work in rats and normal porcine brains. In a further study, they inoculated 13 rats with either green fluorescent protein-labeled U251 cells or epidermal growth factor receptor overexpressing F98 cells, and they imaged brain tumors with FIVE, benchtop confocal microscopy, and standard H\&E staining [54]. Fluorescein isothiocyanate-conjugated EGFR fluorescent antibody (FITC-EGFR) was applied for contrast of F98 cells, while GFP-labeled U251 cells were self-fluorescent. FIVE visualized GFP-labeled tumors as individual satellite cells within peritumoral tissue, a definitive tumor border, and subcellular structures. Both the markers and their tumor regions correlated with standard confocal imaging and histology (Figure 7).

In a recent study contributing to FDA approval of a FIVE system for clinical neurosurgical use, Belykh et al. continued their experiments with the second-generation FIVE imaging and demonstrated that the second-generation FIVE had higher resolution and contrast and offered ease of operation with automated contrast and image metadata analysis [50]. FIVE has also been used in studies of small vascular structures in altered blood flow states, and to assess the characteristics of tumor blood vessels (Figure 8).

Liver. FIVE, with a sterile sheath, has been used for imaging surgically exposed human liver. Although basic features of the microvasculature can be imaged with FIVE and fluorescein staining, histoarchitecture of liver cannot be visualized as effectively due to the thick liver capsule [55]. Hence, a prototype FIVE device with near infrared imaging $(780 \mathrm{~nm})$ was used with indocyanine green as a contrast agent [56]. This follow-up trial overcame the previous challenges and 


\section{Intraoperative results}
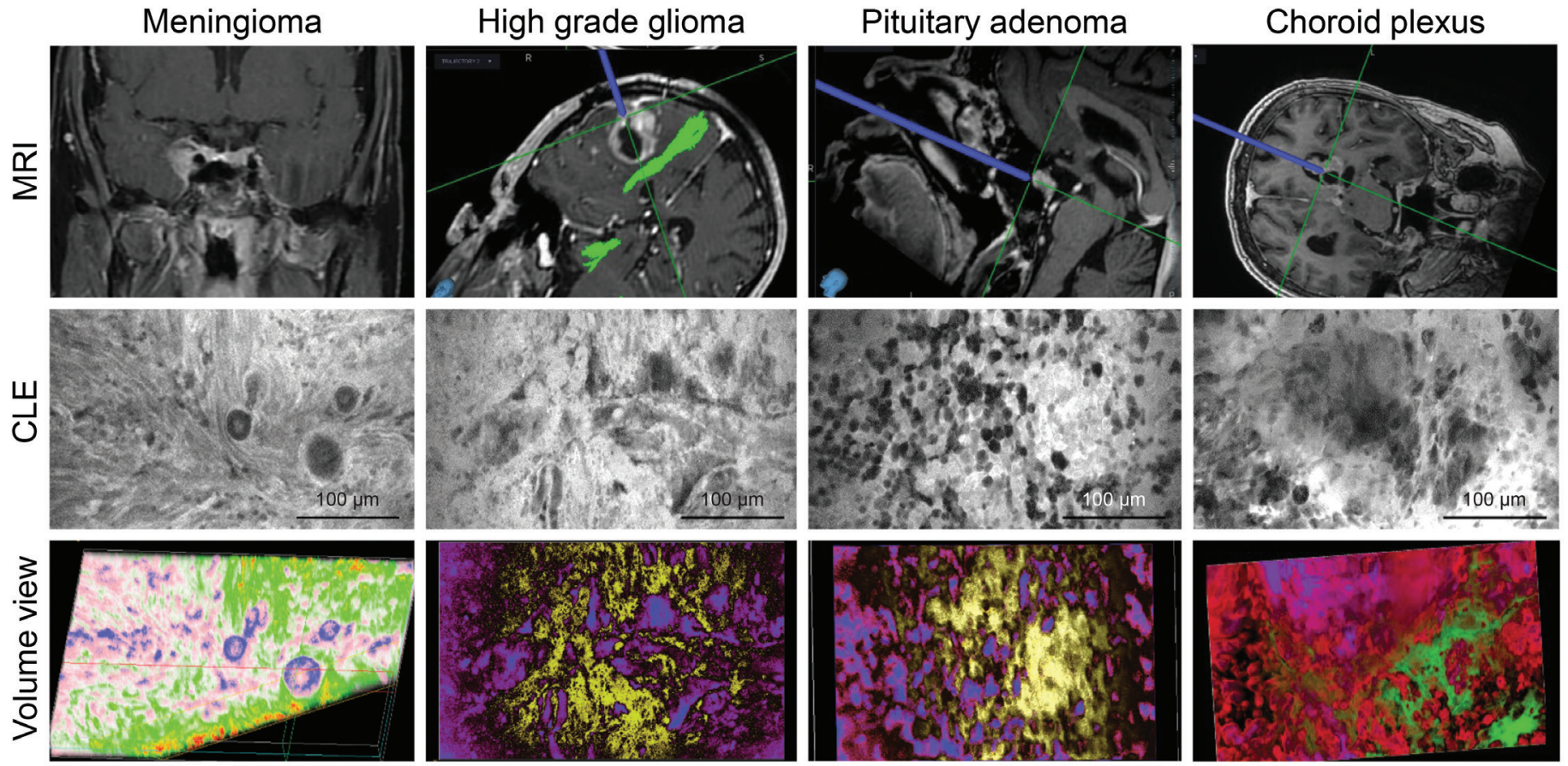

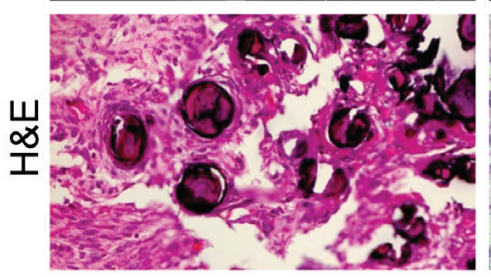

Histological CLE picture showed typical fibrous elongated cells.

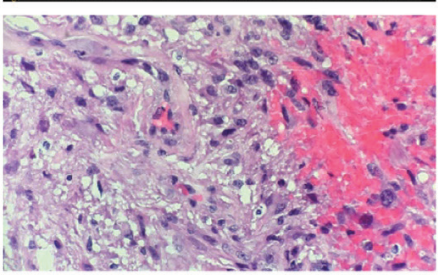

Hypercellular tissue with markedly atypical elongated tumor cells that appeared dark against a bright fluorescent background.

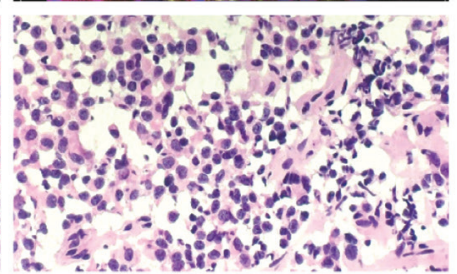

Poorly demarcated nests without septal separation; The tissue appeared densely granulated on the CLE images with monomorphic cells that had irregular staining with fluorescein.

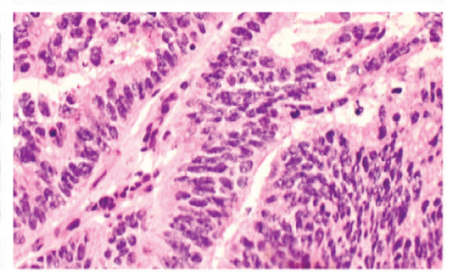

Fusiform tissue architecture, increased cell density, cells arranged in pseudocolumnar fashion. Cells appear anaplastic with pleomorphic nuclei.

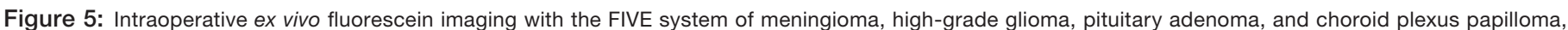

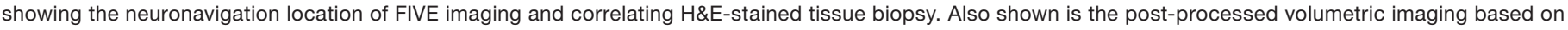
FIVE depth images. Used with permission from Barrow Neurological Institute, Phoenix, Arizona.

revealed liver details similar to those found with traditional histopathology techniques, with the accuracy to predict the presence of steatosis and fibrosis. FIVE has been successfully employed in a study of a murine model of hepatocyte apoptosis. Single hepatocytes were observed for up to 6 hours within the intact liver in a living mouse. This study showed that cells that undergo apoptosis initially swell rather than shrink in the initial phase of apoptosis. Authors also followed cytoplasmic vesicle formation, nuclear condensation, cellular disintegration, and macrophage infiltration. Such procedures and dynamic imaging of cells in their natural environment is not possible ex vivo.

FIVE imaging of other tissues. In a study at Melbourne Royal Women's Hospital, Tan imaged patients undergoing colposcopy for the treatment of low- or high-grade cervical epithelial neoplasia [57]. Different histoarchitectural features of cervix, including squamo-columnar junctions, dermal papillae, endocervical glands, and squamous epithelia were reported. This study also developed a novel scoring system based on the cellular architecture of the tissue and compared the efficiency of FIVE with conventional histology. Based on this scoring system, FIVE was $97 \%$ effective in detecting neoplastic changes in squamous epithelium, $80 \%$ effective in predicting low-grade neoplasia, and $93 \%$ specific in predicting medium- to high-grade neoplasia.

Krishnamurthy developed a simple ex vivo FIVE staining method and yielded results comparable to conventional histopathological examination [58]. This group stained 55 

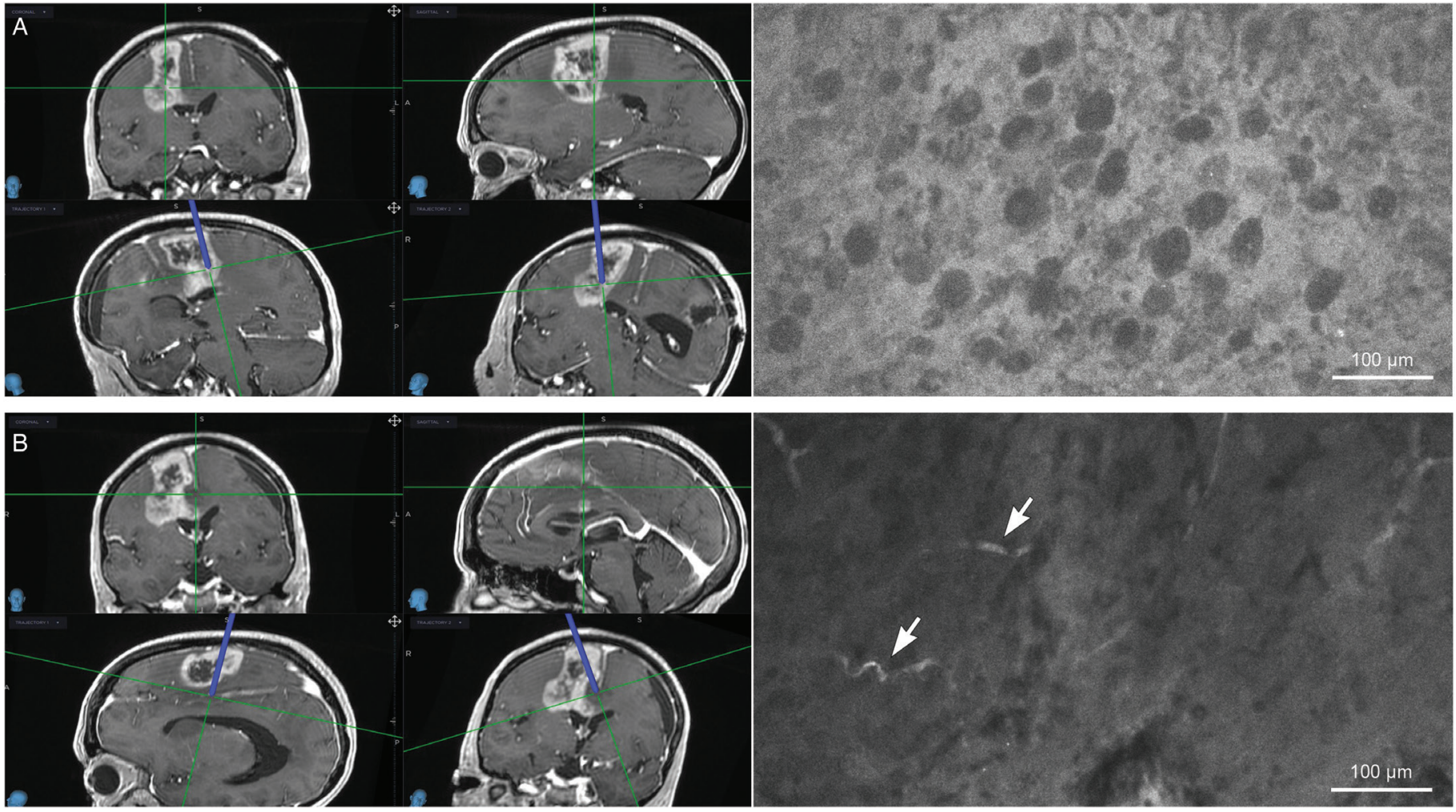

Figure 6: Intraoperative ex vivo FIVE system fluorescein imaging of glioblastoma showing the neuronavigation locations of imaging. FIVE images at locations reveal the (A) aggressive tissue characteristics and (B) irregular capillaries within the malignant tumor mass (arrows). Used with permission from Barrow Neurological Institute, Phoenix, Arizona.

tissue fragments obtained from 16 breast, 18 lung, 14 kidney, and 7 liver surgical excision specimens, stained them with $0.6 \mathrm{mM}$ acridine orange for 6 seconds, and imaged with FIVE before performing standard $\mathrm{H} \& \mathrm{E}$ staining. Acridine orange offered sharp contrast between the nucleus and cytoplasm, recapitulating tissue architecture. Within 5 to 10 minutes, high-quality images were obtained, allowing recognition of the cytomorphologic details for categorization of the imaged tissue similar to histologic examination of H\&E tissue sections. Due to its speed, ease of acquisition, and resolution, authors suggested FIVE's potential in surgical pathology practice.
A

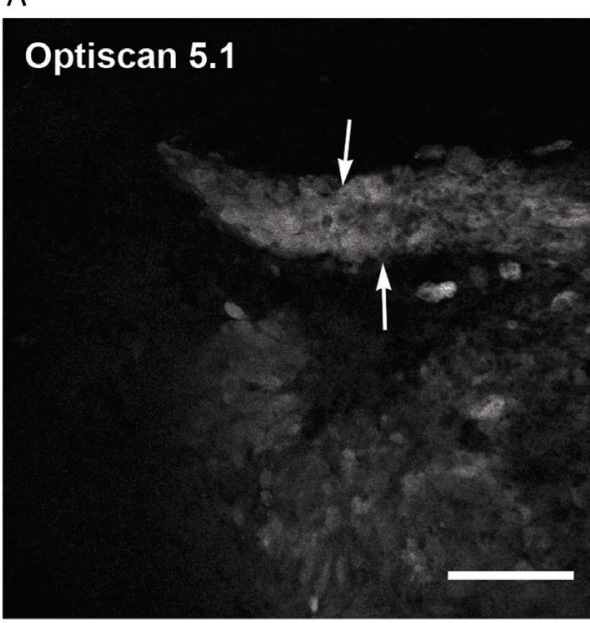

B

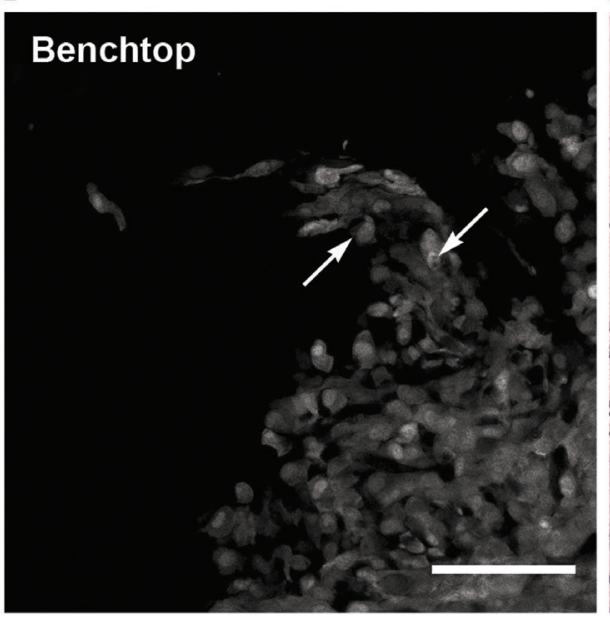

C

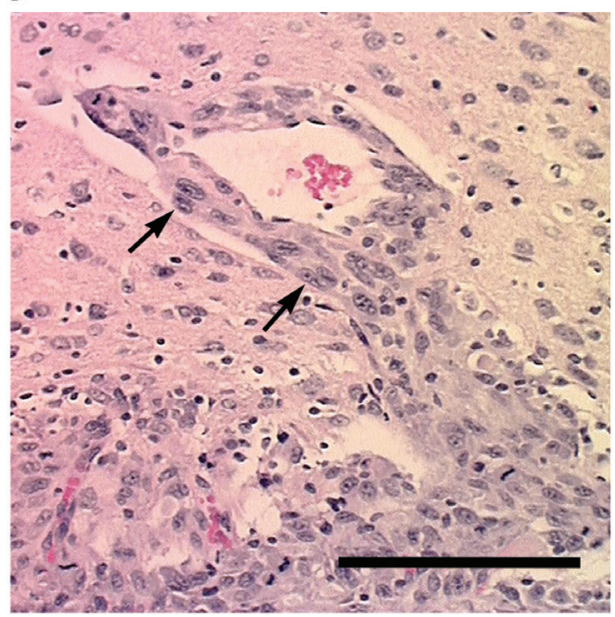

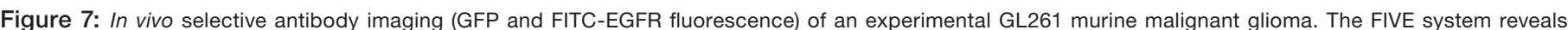

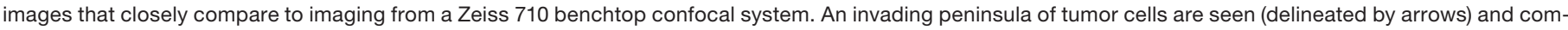

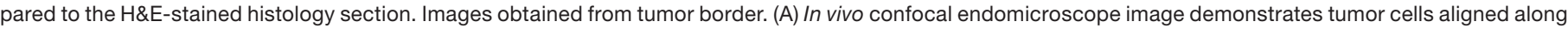

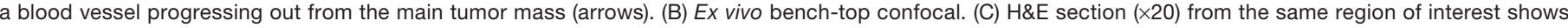
similar histologic features (arrows). Scale bars $=100 \mu \mathrm{m}$. Used with permission from Barrow Neurological Institute, Phoenix, Arizona. 
A

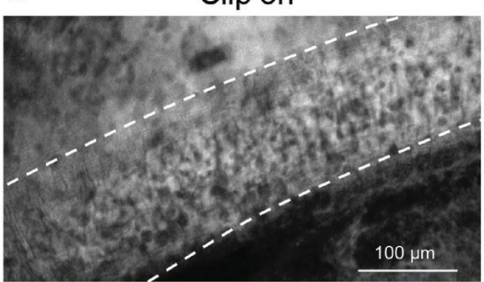

B

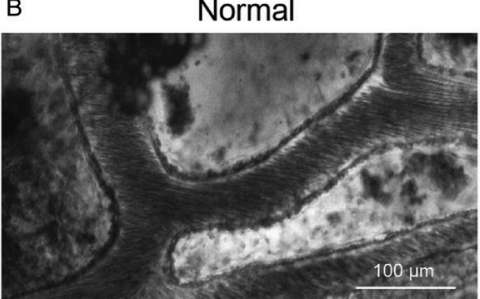

C

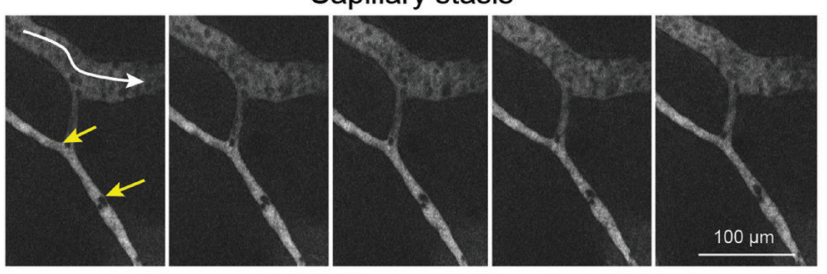

D Vessel constriction and flow redistribution

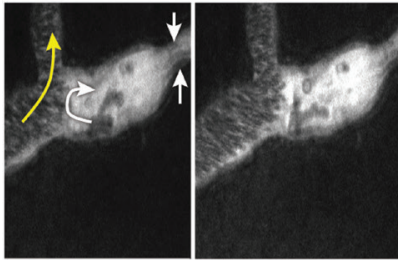

E Normal spinal cord
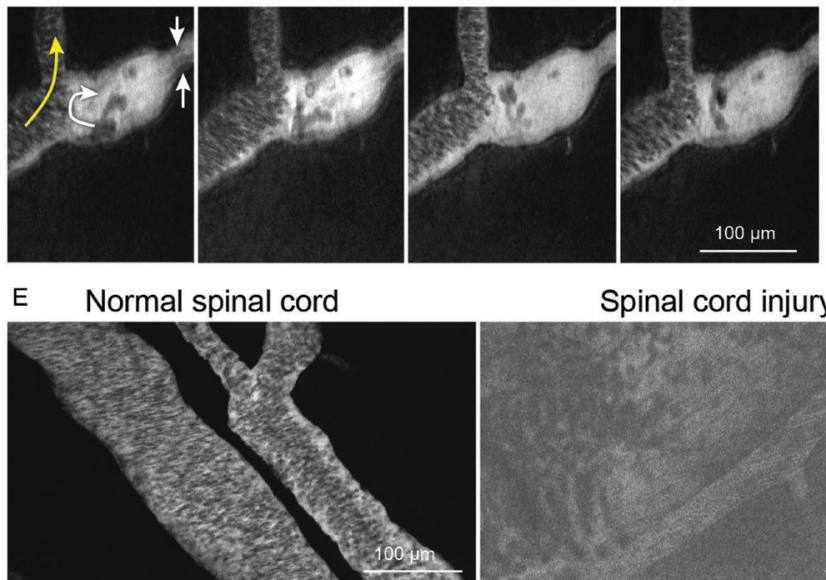

Spinal cord injury
Clip off

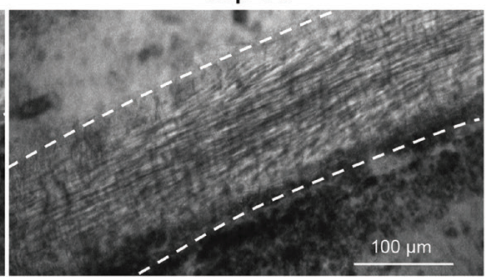

Agglutination
Thrombosis

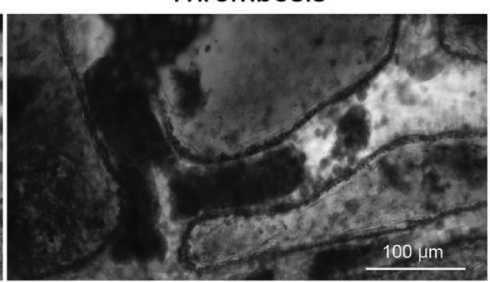

(e)

Brain injury
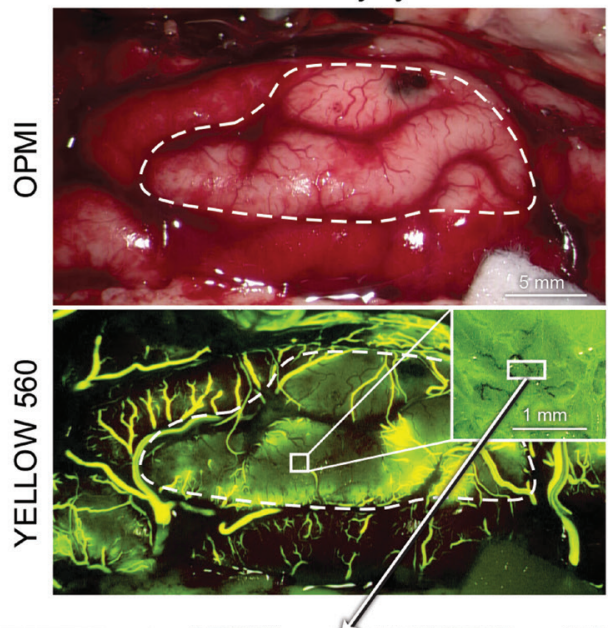
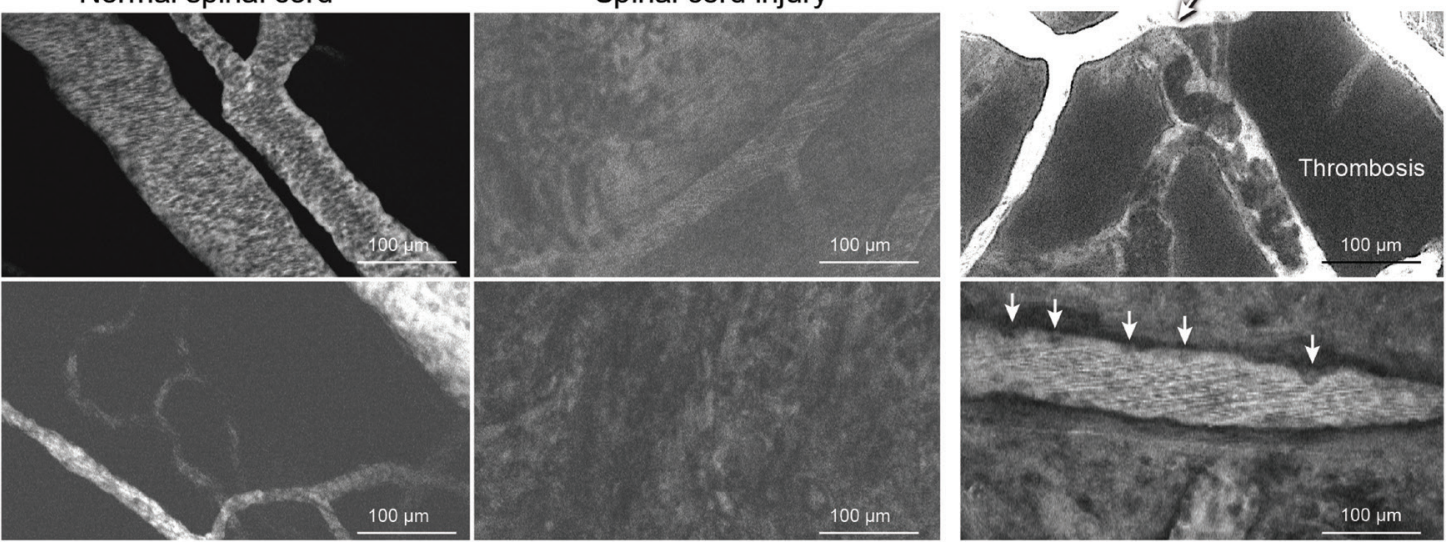

Figure 8: In vivo FIVE fluorescein imaging of blood vessels from swine brain surface. Visualization of the functional state of brain microvasculature in swine. (A) After the clipping of the artery (200-250 $\mu \mathrm{m}$ diameter) proximally (clip on), immediate slowing of the blood flow is observed distally. After the clip is removed (clip off), the restoration of arterial flow pattern is noted. These findings are consistent with repetitive clip application and removal (1 min) in 3 animals. (B) Dynamics of the intraluminal agglutination and thrombi formation in the microvasculature are consistently observed during cardiac arrest at the termination of surgery in all animals. (C) Some collaterals are not functionally active and have stagnant flow (short yellow arrows point to the resting cells in the capillary; long white arrow shows direction of the preserved flow). (D) Constriction (short white arrows) and partial thrombosis of the vessel observed at the area of surgical trauma. The series of images demonstrates that part of the vessel has a normal flow (curved yellow arrow), while part of a vessel has a turbulent flow (curved white arrow) with only a few agglutinated blood cells. (E) Topmost panel shows the cortical microvasculature in the area of cortical injury (cold injury), outlined by the dashed line. In the second panel from the top, a Yellow 560 fluorescent image obtained during the arterial phase shows gross appearance of fluorescein extravasation into the brain tissue. FIVE imaging of the area in the square inset in the right corner is shown in the panel below. In the bottom panel, FIVE shows intraluminal thrombosis in the venules and cell adhesion to the vascular wall (white arrows). Findings are consistent and are observed similarly in areas of cold injury (repeated in 3 animals) and areas of unintentional surgical injury to cortex due to craniotomy (observed in all 5 animals). (F) FIVE images showing uninjured (left panels) and injured (right panels) spinal cord. Normal microvasculature in the spinal cord shows elongated vessels with branches and competent blood-brain barrier preventing contrast extravasation. Multiple extravascular blood cells and extravasation of the contrast are observed at the region of the spinal cord injury. Used with permission from Barrow Neurological Institute, Phoenix, Arizona. 


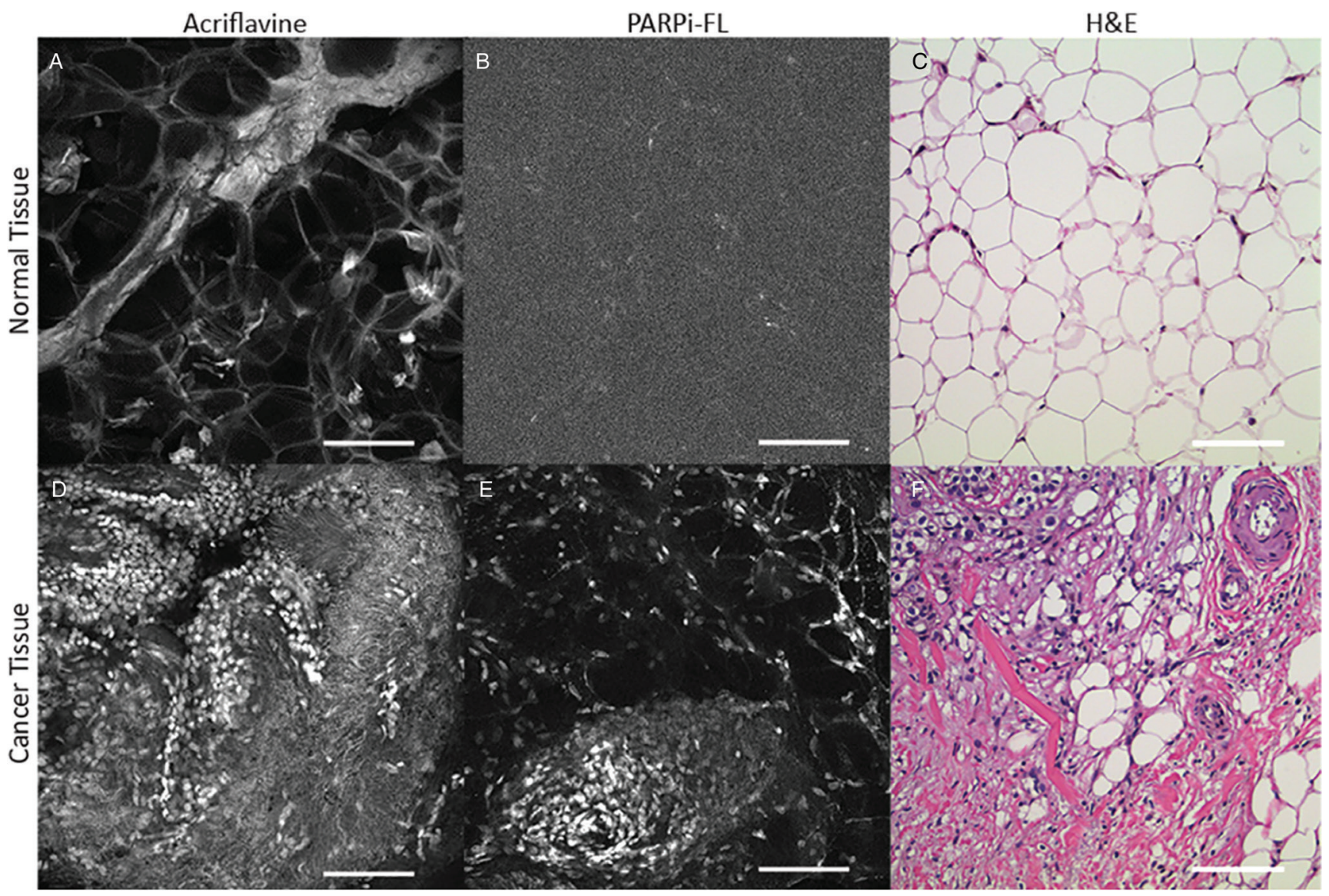

Figure 9: Ex vivo imaging of breast cancer mastectomy specimens using acriflavine, PARPi-FL and matched H\&E histopathology. (A) Acriflavine staining of normal fat and connective tissue. (B) Absence of PARPi-FL staining of normal fat and connective tissue. (C) H\&E staining of normal fat tissue. (D) Acriflavine staining of tumor. (E) PARPi-FL staining of tumor. (F) H\&E staining of tumor. Images courtesy of Dr. Phillip Currie. Scale bars $=100 \mu \mathrm{m}$.

Early detection of tumors and residual tumors after surgery greatly affect tumor recurrence and patient survival. PARP1 inhibitor-based detection of oral tumors has exhibited promising results in preclinical studies [59]. Recent reports also suggest its potential in detecting oral, oropharyngeal, esophageal, and cervical cancers in mice, swine, and human biopsies [60,61]. PARPi-FL has been used to identify tumor in ex vivo breast tissue samples in an unpublished study (Figure 9) and has potential as a breast cancer label in vivo. PARPi-FL was also used successfully in detecting oral cancer when applied as a mouthwash, and discriminated between fresh biopsied samples of the oral tumor and the surgical resection margin with more than $95 \%$ specificity. PARPi-FL, in combination with FIVE imaging, enables a combination of micro and macroscopic evaluation techniques for screening, surveillance, biopsy guidance, fresh biopsy staining, in vivo diagnostic capability, and intraoperative margin delineation [60]. The development of specific fluorescent molecular markers, such as PARPi-FL, offers a range of diagnostic and intraoperative methods that were not previously possible with other imaging modalities.

Zebrafish eyes have also been imaged to demonstrate FIVE's potential to obtain image z-stacks from live animals with good registration, enabling digital reconstruction of the tissue (Figure 10).

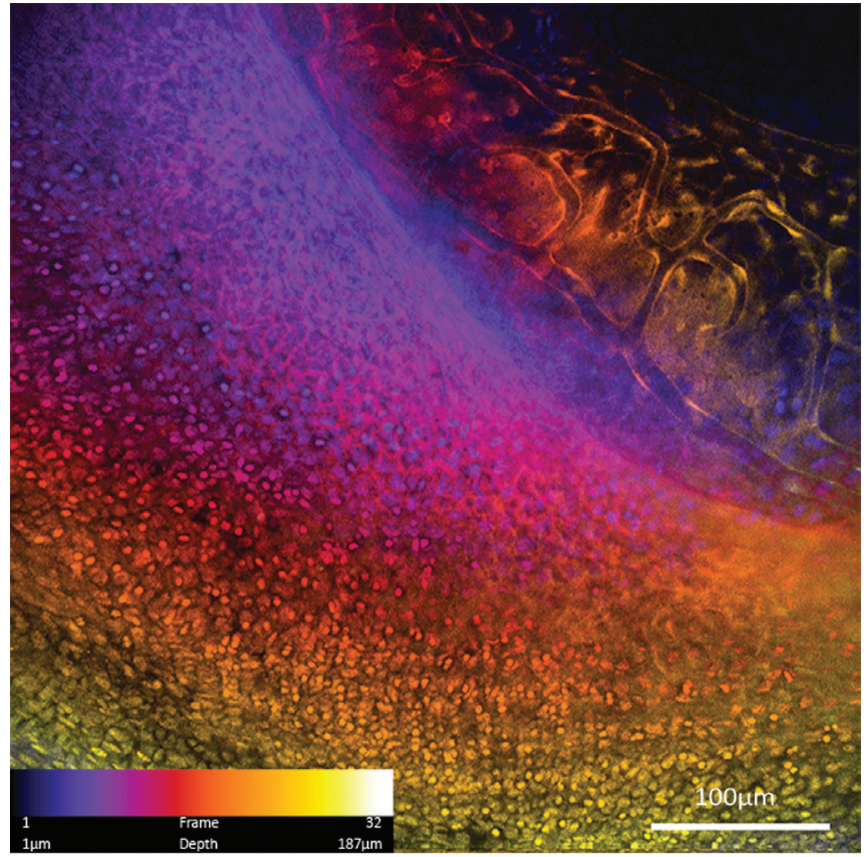

Figure 10: Maximum intensity projection of a $187 \mu \mathrm{m}$ depth color-coded z-stack of zebrafish eye imaged with $488 \mathrm{~nm}$ blue laser scanner. Zebrafish blood vessel endothelium is expressing GFP, and the eye is also stained with acriflavine. 
A Automatic image diagnostic value prediction
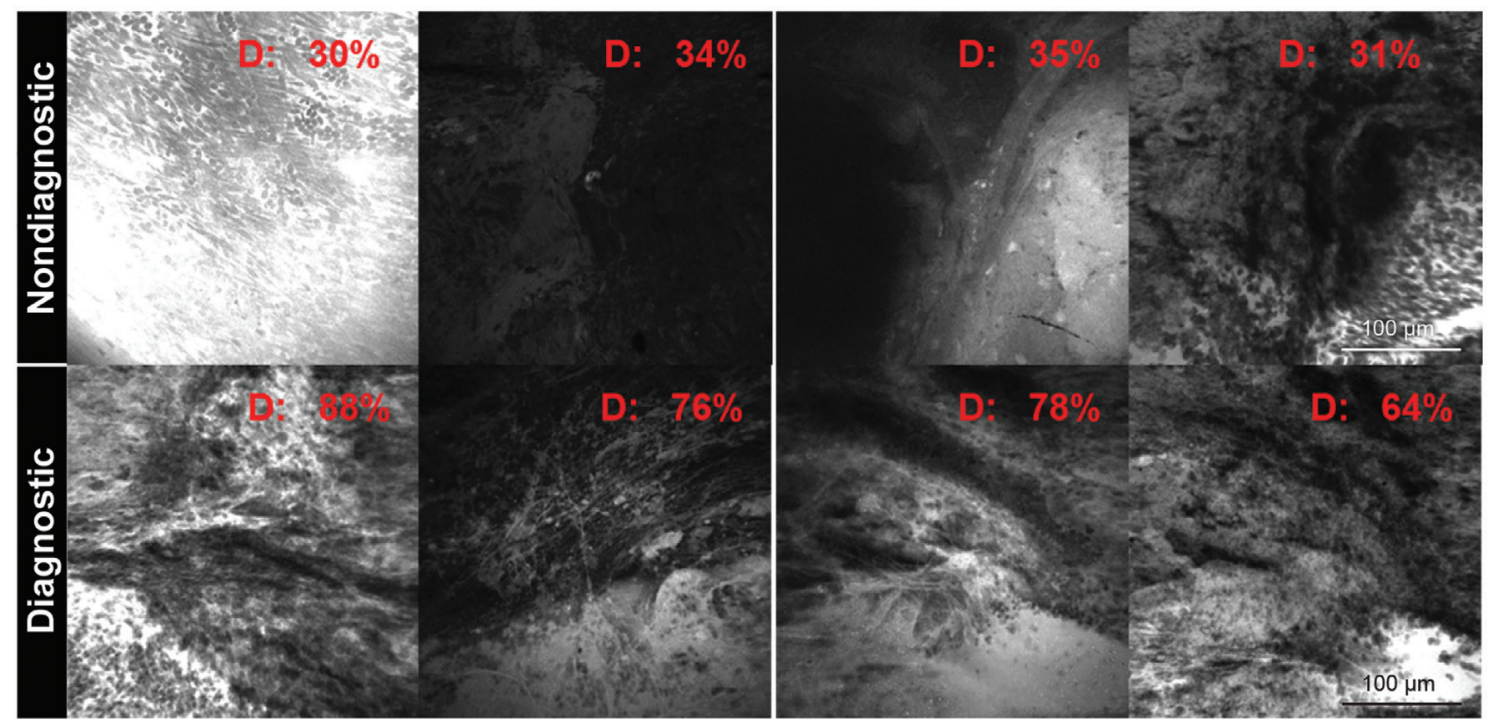

Automatic localization of salient image features from deep neurons
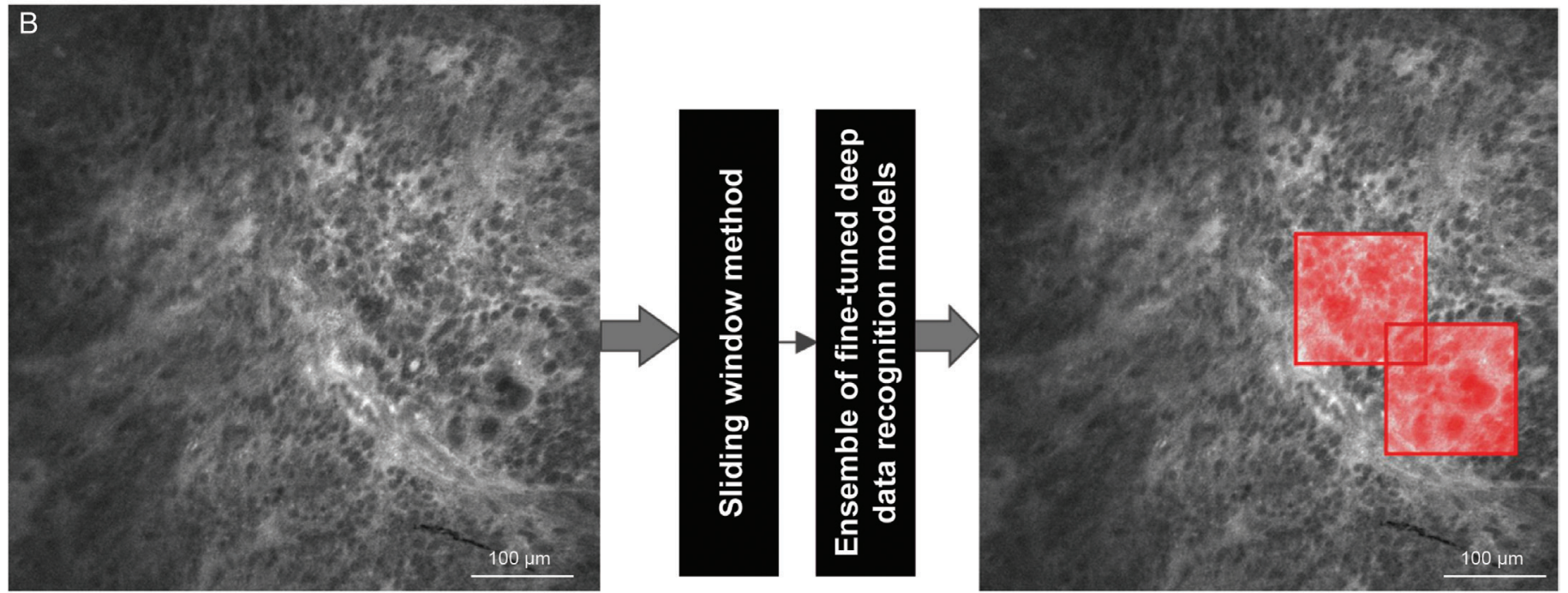

Figure 11: Methods to recognize and discriminate useful regions of FIVE images show that even images that are interpreted as "nondiagnostic" by experienced human raters may, in fact, have some predictive value (A). Often what is or is not useful in an image may be subtle, however, patterns can be rapidly detected after computational system training on a database of images. (B) This rationale is further refined to identify regions or "neurons" within the image that are assessed by algorithms using deep convolutional neural networks that are the most informative, and may yield an important and rapid histological interpretation. Such automated computational image recognition systems will become increasingly crucial to sort useful from non-useful images in clinical confocal endomicroscopic imaging because of the high number of images produced over the course of intraoperative images (about 1 per second). Used with permission from Barrow Neurological Institute, Phoenix, Arizona.

\section{A Joint Future for FIVE and Histopathology}

Despite its high resolution and ability to replace classical histology, FIVE covers a limited field-of-view, and panendomicroscopy of the whole organ is not feasible. Hence, use of FIVE requires a combined approach of macroscopic visualization with targeted FIVE imaging instead of random sampling. In previous studies of chronic ulcerative colitis, FIVE was combined with chromoendoscopy and white light endoscopy $[23,62]$. It showed that the number of biopsies necessary in a conventional colonoscopy group was 42.2, whereas using the combined approach of chromoendoscopy and FIVE only 3.9 biopsies per patient were sufficient. In expert hands, this technique has resulted in the use of endomicroscopy to plan, guide, and survey therapy. FIVE may not replace conventional histopathology, but it serves as an aid to target fewer, smarter biopsies with higher diagnostic yield than large numbers of random untargeted biopsies.

Future perspectives. FIVE provides a cutting-edge tool for real-time histological information in both the basic and clinical sciences. It is translational in its approach as it permits functional imaging, significantly changing our understanding of many diseases. Molecular imaging applications allow detection 


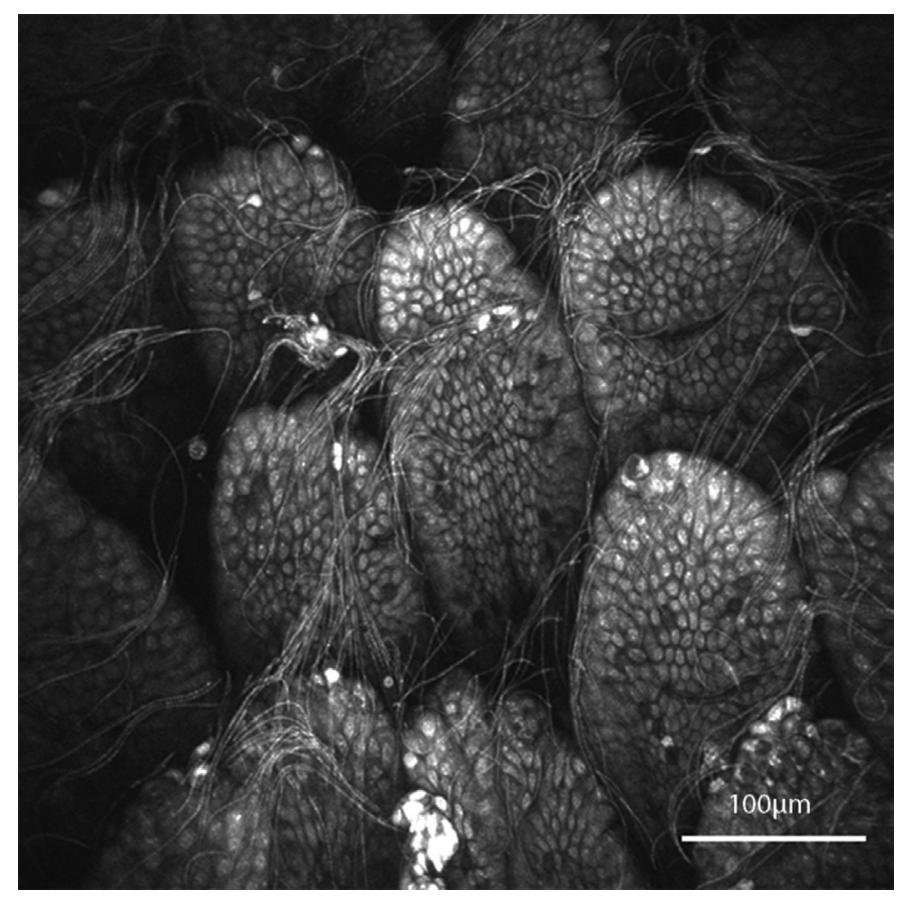

Figure 12: Bacteria in the mucus surrounding microvilli in mouse ileum. Contrast agent, $0.5 \%$ acriflavine applied topically. Image courtesy of Professor Alastair J.M. Watson, MD, FRCP, LRSM Professor of Translational Medicine, University of East Anglia.

and characterization of lesions and may be used for prediction of responses to targeted therapy. Virtual biopsy with FIVE can generate hundreds of images in a short period of time. Hence, deep-learning computational models are proposed for identifying images of interest. Izadyyazdanabadi and others have developed deep-learning algorithms to automatically detect and separate diagnostic images for glioblastoma [63,64] (Figure 11). Such computational models for detecting histological features from FIVE images allow for rapid and detailed diagnosis of diseases. In a further study, these authors developed AlexNet, a deep-learning architecture that can classify images as diagnostic or non-diagnostic [65]. AlexNet was highly reliable when trained on 8,572 nondiagnostic and 8,223 diagnostic images from 74 FIVE-aided brain tumor surgery patients. Findings of these studies have demonstrated that FIVE, when used with AlexNet, can achieve a brain virtual histology model that reliably captures and quickly recognizes diagnostic images. More initiatives are needed to develop AlexNet-like algorithms for other tissue types to increase the efficiency of FIVE.

An interesting FIVE application is in studying the gastrointestinal microbiome (Figure 12). Recent work suggests the interactions between microorganisms significantly affects gastrointestinal, immune system, and brain function. For example, Moussata [32] elucidated the role of intramucosal bacteria in inflammatory bowel disease. Being able to study these interactions in vivo provides a novel tool for investigating this exciting new area of research. Another field where the ability to image cellular interactions in vivo opens new possibilities for understanding is integration of transplanted cells into living organisms. Bergenheim et al. imaged the incorporation of transplanted intestinal stem cells into the gastrointestinal mucosa.

\section{Conclusion}

FIVE enables interrogation of living systems in stunning morphological detail. Advances in fluorophores have provided functional and molecular targeted imaging capabilities that enable the capture of specific cellular events impossible to recreate in vitro. This includes longitudinal studies of physiological and pathological processes and cellular, subcellular, and molecular events in vivo that have not previously been seen. The unexpected nature of some FIVE findings illustrates important roles it can play in the understanding of systems biology, and how it can provide answers to important questions that cannot be visualized by other instruments.

\section{References}

[1] M Rangrez et al., Microscopy Today 29 (2021) https://doi. org/10.1017/S155192952100050X.

[2] NQ Nguyen et al., Ann Surg 249 (2009) https://doi. org/10.1097/SLA.0b013e3181a38f11.

[3] NL Martirosyan et al., Neurosurg Focus 40 (2016) https:// doi.org/10.3171/2016.1.FOCUS15559.

[4] M Goetz et al., Gastroenterology 138 (2010) https://doi. org/10.1053/j.gastro.2009.10.032.

[5] S Foersch et al., Gut 59 (2010) http://dx.doi.org/10.1136/ gut.2009.202986.

[6] AL Polglase et al., Gastrointest Endosc 62 (2005) https:// doi.org/10.1016/j.gie.2005.05.021.

[7] R Kiesslich et al., Best Practice Res Clin Gastroenterol 22 (2008) https://doi.org/10.1016/j.bpg.2008.05.003.

[8] LA Smith et al., J Gastroenterol Hepatol 23 (2008) https:// doi.org/10.1111/j.1440-1746.2008.05463.x.

[9] A Tan and F Macrae, Journal of Gastroenterology and Hepatology 26 (2011) https://doi.org/10.1111/j.1440-1746. 2011.06768.x.

[10] P Wang et al., World J Gastroenterol 16 (2010) https:// dx.doi.org/10.3748/wjg.v16.i41.5203.

[11] R Kiesslich et al., Gastroenterol 128 (2005) https://doi. org/10.1053/j.gastro.2004.12.035.

[12] YT Guo et al., Endoscopy 40 (2008) https://doi.org/ 10.1055/s-2007-995633.

[13] WB Li et al., Surg Endosc 24 (2010) https://doi.org/10.1007/ s00464-009-0608-y.

[14] LK Wanders et al., Lancet Oncol 14 (2013) https://doi. org/10.1016/S1470-2045(13)70509-6.

[15] Z Liu et al., Surg Endosc 33 (2019) https://doi.org/10.1007/ s00464-018-6519-z.

[16] C Trovato et al., Digestive Liver Dis 45 (2013) https://doi. org/10.1016/j.dld.2012.12.016.

[17] KB Dunbar et al., Gastrointest Endosc 70 (2009) https:// doi.org/10.1016/j.gie.2009.02.009.

[18] MI Canto et al., Gastrointest Endosc 79 (2014) https://doi. org/10.1016/j.gie.2013.09.020.

[19] K Venkatesh et al., Gastrointest Endosc 75 (2012) https:// doi.org/10.1016/j.gie.2011.11.013.

[20] EC Gorospe et al., Gastrointest Endosc 76 (2012) https:// doi.org/10.1016/j.gie.2012.07.005.

[21] R Kiesslich et al., Clin Gastroenterol Hepatol 4 (2006) https://doi.org/10.1016/j.cgh.2006.05.010.

[22] Y Iguchi et al., J Gastroenterol Hepatol 24 (2009) https:// doi.org/10.1111/j.1440-1746.2009.05892.x. 
[23] M Goetz et al., Nature Rev Gastroenterol Hepatol 11 (2014) https://doi.org/10.1038/nrgastro.2013.134.

[24] WB Li et al., Gut 60 (2011) http://dx.doi.org/10.1136/ gut.2010.223586.

[25] R Ji et al., Surg Endosc 25 (2011) https://doi.org/10.1007/ s00464-010-1490-3.

[26] MJ Sharman et al., J Vet Internal Med 28 (2014) https://doi. org/10.1111/jvim.12332.

[27] RWL Leong et al., Gastroenterol 135 (2008) https://doi. org/10.1053/j.gastro.2008.08.054.

[28] R Kiesslich et al., Gastroenterol 133 (2007) https://doi. org/10.1053/j.gastro.2007.09.011.

[29] W Dolak et al., Surg Endosc 30 (2016) https://doi. org/10.1007/s00464-015-4572-4.

[30] K Rieger et al., Hematol Oncol 36 (2017) https://doi. org/10.1002/hon.2446.

[31] C Trovato et al., Digestive Liver Dis 41 (2009) https://doi. org/10.1016/j.dld.2008.09.019.

[32] D Moussata et al., Gut (60) 2011 http://dx.doi.org/10.1136/ gut.2010.213264.

[33] CQ Li et al., Amer J Gastroenterol 105 (2010) https://doi. org/10.1038/ajg.2009.664.

[34] VC Wasinger et al., Sci Rep 10 (2020) https://doi. org/10.1038/s41598-020-69746-w.

[35] DP Hurlstone et al., Brit J Surg 95 (2008) https://doi. org/10.1002/bjs.5988.

[36] R Kiesslich et al., Gastroenterol 127 (2004) https://doi. org/10.1053/j.gastro.2004.06.050.

[37] S Sanduleanu et al., Clin Gastroenterol Hepatol 8 (2010) https://doi.org/10.1016/j.cgh.2009.08.006.

[38] H Bao et al., J Biomed Optics 14 (2009) https://doi. org/10.1117/1.3269681.

[39] M Goetz et al., Gastrointest Endosc 70 (2009) https://doi. org/10.1016/j.gie.2009.04.016.

[40] G Bari et al., Turkish J Surg 34 (2018) https://doi. org/10.5152/turkjsurg.2018.4181.

[41] T Kovács et al., Shock 38 (2012) https://doi.org/10.1097/ SHK.0b013e31825d1ed0.

[42] G Varga et al., Inflammopharmacology 26 (2018) https:// doi.org/10.1007/s10787-017-0354-z.

[43] G Vargas et al., Antimicrob Agents Chemother 60 (2016) https://doi.org/10.1128/AAC.00134-16.

[44] BR Haxel et al., Eur Arch Oto-Rhino-Laryngol 267 (2010) https://doi.org/10.1007/s00405-009-1035-3.

[45] AI Musani et al., J Bronchol Intervent Pulmonol 17 (2010) https://doi.org/10.1097/LBR.0b013e3181da2f98.

[46] GR Bernard et al., Am J Respir Critical Care Med 149 (1994) https://doi.org/10.1164/ajrccm.149.3.7509706.

[47] MI Schwarz and RK Albert, Chest 125 (2004) https://doi. org/10.1378/chest.125.4.1530.

[48] F Chagnon et al., Lab Invest 90 (2010) https://doi.org/ 10.1038/labinvest.2010.76.

[49] O Lesur et al., J Clin Med 8 (2019) https://doi.org/ doi: $10.3390 / j \mathrm{~cm} 8081197$.

[50] E Belykh et al., Cancer Management Res 10 (2018) https:// doi.org/10.2147/CMAR.S165980.

[51] T Sankar et al., Neurosurgery 66 (2010) https://doi.org/ 10.1227/01.NEU.0000365772.66324.6F.
[52] M Peyre et al., Brain Pathol 23 (2013) https://doi. org/10.1111/bpa.12039.

[53] KE Fenton et al., Neurosurg Focus 36 (2014) https://doi. org/10.3171/2013.12.FOCUS13488.

[54] NL Martirosyan et al., Surg Neurol Intl 7 (2016) https:// doi.org/10.4103/2152-7806.195577.

[55] M Goetz et al., Endoscopy 40 (2008) https://doi.org/ 10.1055/s-2008-1077296.

[56] M Goetz et al., J Hepatol 53 (2010) https://doi.org/10.1016/ j.jhep.2010.01.039.

[57] J Tan et al., BJOG Intl J Obstet Gynaecol 116 (2009) https:// doi.org/10.1111/j.1471-0528.2009.02261.x.

[58] S Krishnamurthy et al., Arch Pathol Lab Med 142 (2018) https://doi.org/10.5858/arpa.2017-0164-OA.

[59] P Demétrio de Souza França et al., Sci Rep 10 (2020) https://doi.org/10.1038/s41598-020-67958-8.

[60] S Kossatz et al., Nature Biomed Eng 4 (2020) https://doi. org/10.1038/s41551-020-0526-9.

[61] S Kossatz et al., Sci Rep 6 (2016) https://doi.org/10.1038/ srep21371.

[62] R Kiesslich et al., Gastroenterol 132 (2007) https://doi. org/10.1053/j.gastro.2007.01.048.

[63] M Izadyyazdanabadi et al., J Vis Commun Image Represent 54 (2018) https://doi.org/10.1016/j.jvcir.2018.04.004.

[64] M Izadyyazdanabadi et al., Front Oncol 8 (2018) https:// doi.org/10.3389/fonc.2018.00240.

[65] M Izadyyazdanabadi et al., Proc SPIE Med Imaging 101342J (2017) https://doi.org/10.1117/12.2254902.

\section{MT}

\section{SEM Scintillators \& Light Guides} Light Guide Recoating Services too!

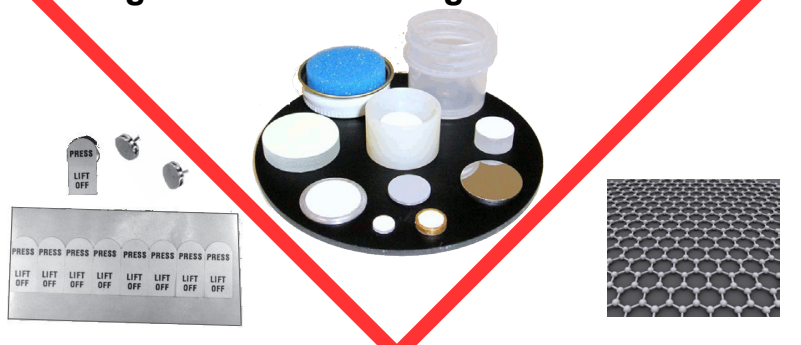

Conductive Adhesives, Introducing GRAPHENE Coatings \& Tabs!

The New Carbon!

Brush or Spray the same product!

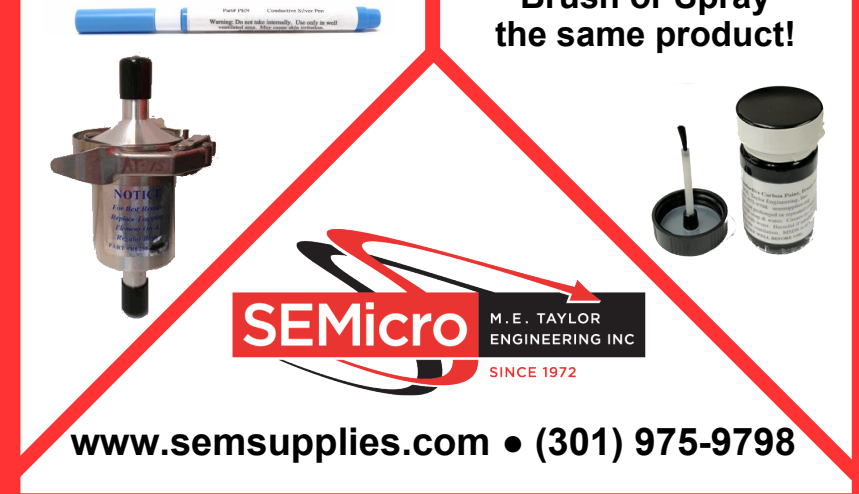


Radiation hard

\section{unsaturated \\ Zero Loss Peak}

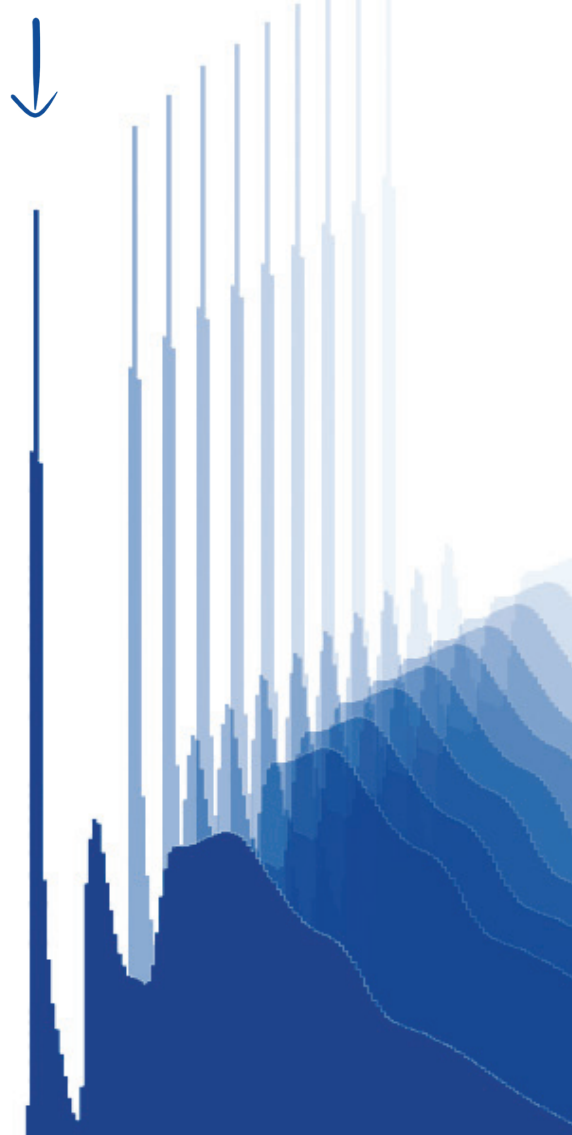

High dynamic range

over seven orders of magnitude

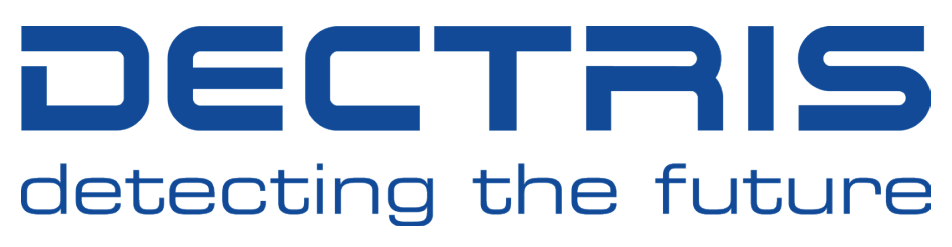

The highest frame rate

up to 18.000 frames

per second

Noise-free detection

single-electron counting across the whole spectrum

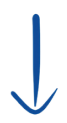

Make the most of electron microscopy with hybrid-pixel detectors

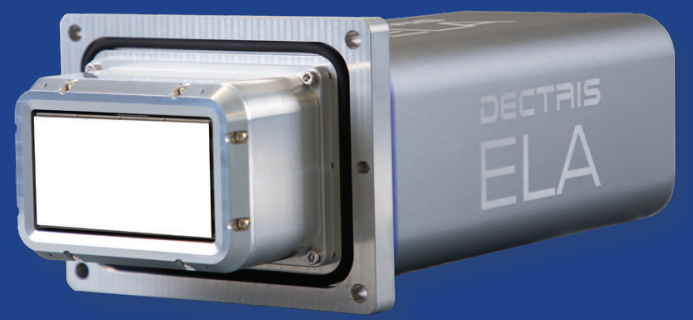

